\title{
DYNAMICS OF RAT ENDOMETRIUM AS STUDIED WITH SEM AND VASCULAR CORROSION CASTS.
}

\author{
Udo M. Spornitz and Irena Bartuskova
}

\author{
Dept. of Electron Microscopy, Institute of Anatomy, University of Basel, CH 4056 Basel, \\ Switzerland,
}

The rat is known to have a relatively short endometrial cycle which lasts only about four days. The cycle comprises proestrus, estrus, diestrus I, and diestrus II. Every stage lasts for about 24 hours. Towards the end of the rats life span the cycle may increase to about 6 days. During each of the individual stages characteristic morphological features are present which allow easy identification [1]. One of the outstanding features of estrus e.g. is the presence of pseudoglands, i.e. numerous depressions (pits) of the surface epithelium which resemble glandular orifices (fig. 1). They are formed through necrosis and apoptosis of the endometrial epithelium, they do not however penetrate the underlying basallamina, thus forming a cul de sac. Apoptosis of the involved cells can clearly be detected on the basis of nuclear fragmentation and the marginalization of chromatin. In the necrotic cells giant lysosomal vesicles are formed (fig. 2). The digestion of necrotic and apoptotic cells causes the endometrial surface epithelium to sink below the level of the surrounding cells thus causing the pits or pseudoglands. - Scanning electron microscopic detection of alkaline phosphatase shows a maximum amount during estrus and a minimum during diestrus II [2]. During early pregnancy the alkaline phosphatase activity decreases to zero on day 5 i.e. the actual day of blastocyst adhesion [3]. During the immediate postpartum period again the alkaline phosphatase activity is very low and the rat could become pregnant again. This stage is called postpartum diestrus. Shortly after this stage has been passed the rat then enters postpartum estrus, during which the alkaline phosphatase activity becomes rather high again. This in turn goes along with the fact that the rat could not become pregnant. The absence or presence of alkaline phosphatase activity could thus be taken as a marker for the relative receptivity of the animal. - Despite of the short cycle of the rat, the vascular pattern of the endometrial vessels also changes considerably as investigated with corrosion cast methods (fig. 3). Particular the capillary network seems to be constantly remodeled from one cycle day to the next. One of the outstanding characteristics of the immediate subsurface capillaries of the rat endometrium is that they change their diameter during the course of the cycle. Thus the average diameter measures about $18 \mu \mathrm{m}$ during diestrus II and less than $10 \mu \mathrm{m}$ during proestrus. For this three possible mechanisms could be responsible: 1 . The capillary network is regulated through precapillary sphincters. 2 . The capillary network is in fact constantly remodeled. 3 . The diameter is controlled through the action of pericytes.- On the corrosion casts we could only find impressions from precapillary sphincters in connection with deeper capillaries and not with the immediate subsurface capillaries. This would in fact imply that the immediate subsurface capillaries are either remodeled or that their diameter is perhaps controlled through a large number of pericytes.

[1] U .M. Spornitz, et al. 1999, Anat. Rec. 254: 116 - 126

[2] U. M. Spornitz, et al. 1997, Recent Advances in Microscopy of Cells Tissues and Organs, Pietro Motta ed. Antonio Delfino Publisher, Rome 553 - 560

[3] A. Winkelmann and U.M. Spornitz 1997, Acta Anat. 158: 237 - 246 

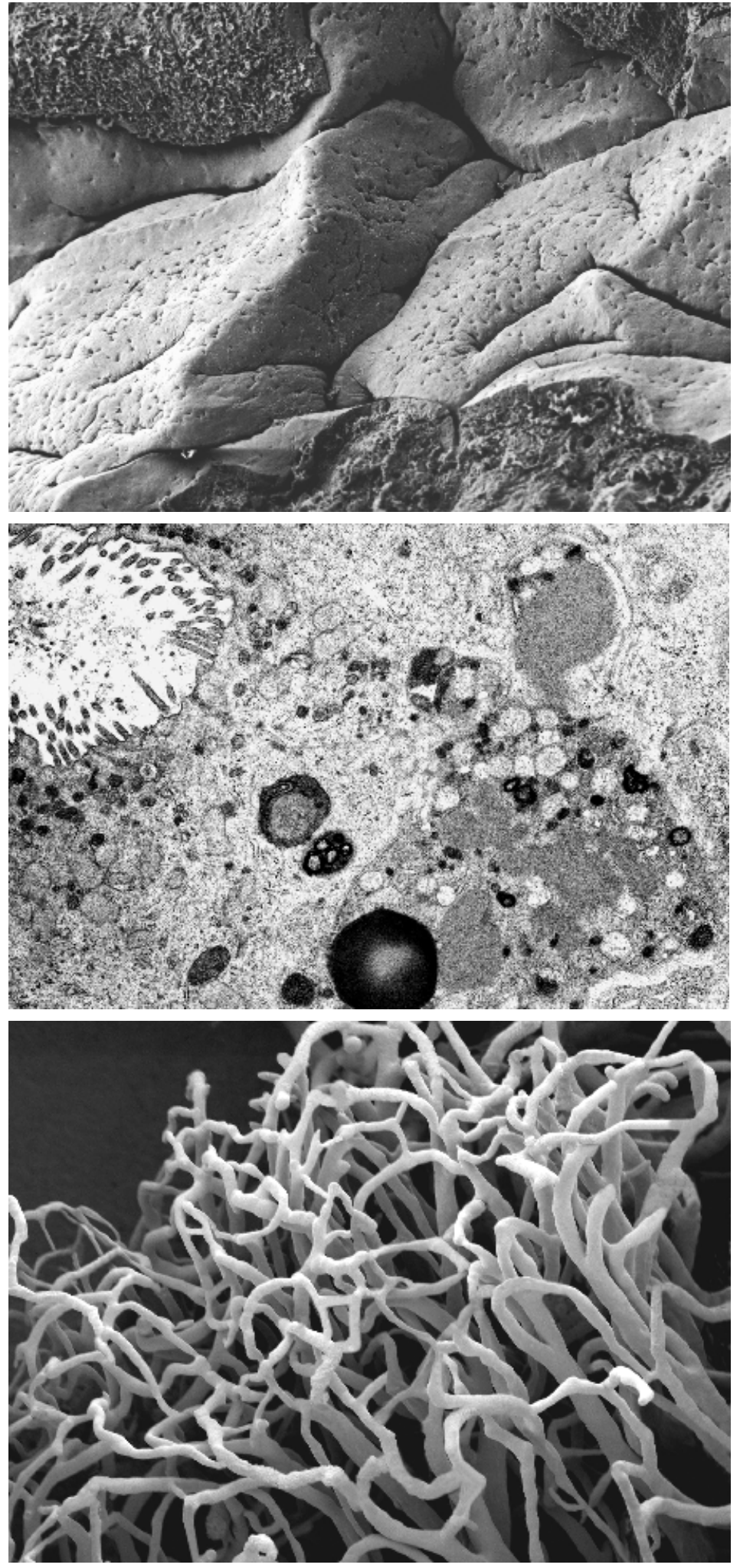

Fig. 1

Low power SEM micrograph of the luminal surface of the endometrium during estrus with folds and ridges like they are typical for this cycle stage. The many depressions are "pseudoglands", i.e. pits formed through necrosis and apoptosis of the underlying cells.

Fig. 2

Transmission electron micrograph which shows the deepest part of a depression in the upper left corner. These "pseudoglands" are formed through necrosis and apoptosis. Large parts of this micrograph is filled with an autophagic vacuole, which contains nuclear fragments among other more or less digested cellular organelles.

Fig. 3

Corrosion cast of the capillary network during diestrus II. Note the many interconnections between the immediate subsurface capillaries. These are much smaller in diameter than the capillaries of deeper layers. 